\title{
Presenilin 1 transgene addition to amyloid precursor protein overexpressing transgenic rats increases amyloid beta 42 levels and results in loss of memory retention
}

\author{
Cansu Agca ${ }^{1}$, Diana Klakotskaia ${ }^{2}$, Todd R. Schachtman², Anthony W. Chan ${ }^{3}$, James J. Lah ${ }^{4}$ and Yuksel Agca ${ }^{\text {* }^{*}}$
}

\begin{abstract}
Background: We previously reported the production of transgenic rats (APP21 line) that over-express human amyloid precursor protein (APP) containing Swedish and Indiana mutations. In order to generate a better model for Alzheimer's disease (AD), the APP21 rat line was used to generate double transgenic line that over-expressed Presenilin 1 (PS1) with L166P mutation in addition to APP transgene (APP + PS1 line).

Results: Thirty-two double transgenic founders were generated and the ultimate transgenic founder was selected based on PS1 transgene copy number and level of amyloid-beta $(A \beta)_{42}$ peptide. The APP + PS1 double transgenic rats had 38 times more PS1 in brains compared to APP rats. Behavioral assessment using Barnes maze showed that APP + PS1 rats exhibited a larger learning and memory deficit than APP21 rats. Double transgenic rats also produced more $A \beta_{42}$. Histological examination of the brains showed that the APP21 rat line displayed neurofibrillary tangles and in contrast, the APP + PS1 line showed chromatolysis in hippocampal neurons and neuronal loss in CA3 region of hippocampus.
\end{abstract}

Conclusions: Due to the separate segregation of APP and PS1 transgenes in APP + PS1 double transgenic rats, this transgenic line may be a valuable model for studying the effects of various levels of APP and PS1 transgenes on various aspects of brain pathologies associated with the AD phenotype.

\section{Background}

Alzheimer's disease (AD) is one of the most devastating and costly diseases that affect approximately $12 \%$ of the population over the age of 65 [2]. Alzheimer's disease is associated with the over-production and reduced clearance of amyloid-beta (A $\beta)$ peptides [19]. Cleavage of amyloid- $\beta$ precursor protein (APP) by the presenilin (PS) 1 enzyme results in the release of $A \beta$ peptides, which aggregate and form $A \beta$ plaques in the brain. These $A \beta$ plaques and neurofibrillary tangles are the two primary pathological manifestations of $\mathrm{AD}$ in the brain.

\footnotetext{
*Correspondence: agcay@missouri.edu

1 Department of Veterinary Pathobiology, College of Veterinary Medicine, University of Missouri, 1600 East Rollins Street, Room W191, Columbia, MO 65211, USA

Full list of author information is available at the end of the article
}

Amyloid peptides are produced via the cleavage of APP by $\Upsilon$-secretase, a protease complex of four proteins that includes PS1 or PS2, which are both homologous proteins that contain the catalytic site of the enzyme [7]. $\Upsilon$-secretase cleaves the $\beta$-amyloid protein into two major forms of $A \beta$ polypeptides, $A \beta_{40}$ and $A \beta_{42}$. The relative amount of the longer form of $A \beta, A \beta_{42}$, is particularly critical for $A D$ progression because it is more prone to aggregation than the shorter $A \beta_{40}$ peptide $[4,10,23]$. In addition, the ratio of $A \beta_{42} / A \beta_{40}$ correlates with the load of $A \beta$ plaques in the brain $[8,14]$. Furthermore, the reduction of PS1 mRNA using siRNA against PS1 reduced $A \beta_{42}$ in cultured cells [11] which suggests that lower levels of PS1 lead to decreased production of A $\beta_{42}$.

We previously generated rats that overexpress human APP containing the Swedish and Indiana mutations [1]. 
The APP21 line expressed high levels of APP, but failed to produce $A \beta$ plaques in the brain. However, intrahippocampal seeding of dilute Alzheimer brain extracts containing aggregated $A \beta$ led to the production of plaques in APP21 rats [20]. This finding indicates that plaque formation depends on environmental challenges, conditions which laboratory rats are not exposed to routinely. In order to increase the pathogeny of overexpressed APP, we generated double transgenic rats from the APP21 line that over-express both the PS1 and the APP transgenes. Our hypothesis was that the addition of a PS1 transgene would increase more toxic $A \beta_{42}$ polypeptide and thus, would produce a more suitable model for examining AD.

\section{Methods}

Animals, construct design, and preparation of vesicular stomatitis virus glycoprotein pseudotyped lentivirus

The homozygous APP21-transgenic rat line [1], containing human APP with the Swedish double missense mutation (K595M/N596L) and Indiana single missense mutation (V642F), were used for the generation of APPPS1 double transgenic rats. Inbred Fischer 344 rats were used as the background strain for the APP21 rat line. The human PS1 cDNA coding region corresponding to 2851688 bases of PS1 variant 1 (GenBank Accession number NM_000021.3) with L166P mutation was cloned into the Lentivial vector ( $\mathrm{pLV}$ ) containing ubiquitin-C (Ubi-C) and enhanced green fluorescence protein (eGFP; pLVU-eGFP) cassette [17] in place of the eGFP coding sequence. The new vector was designated as PLVU-PS1. The Ubi-C promoter, which is upstream of PS1, controlled PS1 transcription. The pLVU-PS1 was a self-inactivating vector, composed of the woodchuck hepatitis virus posttranscriptional regulatory element (WRE) to increase transcription levels and minimize position effects. Additionally, a human immunodeficiency virus-1-flap element was inserted between the $5^{\prime}$ long terminal repeat and the internal promoter, which increased the titer. The preparation of vesicular stomatitis virus glycoprotein pseudotyped Lentivirus was as described by Lois et al. [17]. All animal studies were performed in accordance with the University of Missouri's Animal Care and Use Committee guidelines and the Institute for Laboratory Animal Research Guide for the Care and Use of Laboratory Animals. The rats were housed in conventional cages at $20-25^{\circ} \mathrm{C}$ in a controlled lighting environment and provided free access to water and standard pellet rodent chow.

\section{Zygote collection, microinjection of lentiviral vector, and embryo transfer}

Homozygous APP21-transgenic female rats (28-30 dayold) were super ovulated by administering intraperitoneal (IP) injections of 15 IU pregnant mares' serum gonadotropins (PMSG) followed by IP injections of 15 IU human chorionic gonadotropin $52 \mathrm{~h}$ after PMSG. Twenty-four hours after mating, rats were euthanized using $\mathrm{CO}_{2}$ and zygotes were collected from the oviducts. Morphologically normal zygotes having two pronuclei and a sperm tail were used for lentiviral vector injections into perivitelline space as described earlier [1]. Lentiviral vector-injected zygotes were then transferred into the oviducts of 8-10 week-old Sprague-Dawley pseudopregnant recipient rats.

\section{Genomic DNA isolation and polymerase chain reaction}

Genomic DNA from tail-snips was isolated using the Wizard Genomic DNA Purification Kit (Promega, Madison, WI). Screening of transgenic rats was conducted by PCR using primers annealing the Ubi-C promoter (Forward; TGTCCGCTAAATTCTGGCCGTT) and PS1 transgene (Reverse; AGCATTCAGAATTGAGTGCAGGGC). The PCR reactions were carried out using $50 \mathrm{ng}$ genomic DNA, 100 ng of each primer and 1.5 U Biolase taq (Bioline, Randolph, MA), and amplified PCR products were size separated through a $1 \%$ agarose gel stained with ethidium bromide for visualization.

\section{ELISA measurement of $A \beta_{40}$ and $A \beta_{42}$}

Serum samples were analyzed for $A \beta_{40}$ and $A \beta_{42}$ using commercial ELISA kits (Genetics Company, Schlieren, Switzerland). Serum samples were diluted in assay buffer and processed according to the manufacturer's recommended protocols. Briefly, samples and standards were incubated in capture wells overnight at $4{ }^{\circ} \mathrm{C}$ with either a biotinylated $A \beta_{40}$ or an $A \beta_{42}$-specific antibody. After several rinses, the enzyme-conjugated detection reagent was added to the wells for $30 \mathrm{~min}$. After additional rinses, wells were incubated with the chromogen solution for $30 \mathrm{~min}$ at room temperature and were shielded from light. After the addition of the stop solution, the wells were read for absorption at $450 \mathrm{~nm}$ and the $A \beta$ concentration in the samples was calculated based on standard curves.

\section{Southern blot analysis}

Southern blot analysis was conducted to determine the copy number of the transgene in founder rats. Genomic DNA was digested with EcoRI, which cut the junction of the PS1 and the WRE transgene. The digestion products were size-separated through a $0.8 \%$ agarose gel and transferred to a Genescreen plus membrane (Perkin Elmer, Wellesley, MA) overnight. The 524 bp PS1 probe template was prepared by amplification of pLVU-PS1 using forward (AGGTCCACTTCGTATGCTGGTTGA) and reverse (TGATGGAGATTGGAAGAGTGGCA) primers. The ${ }^{32} \mathrm{P}$ labeled probe was generated using the probe 
template, Ready-To-Go DNA Labeling Beads (Amersham Biosciences, Piscataway, NJ) and $\left[\alpha-{ }^{32} \mathrm{P}\right]-\mathrm{dCTP}$ (Perkin Elmer, Wellesley, MA). The membranes were prehybridized in $10 \%$ dextran sulfate, $6 \times$ saline sodium sitrate (SSC), $1 \%$ sodium dodecyl sulfate (SDS) for $2 \mathrm{~h}$ and hybridized using the ${ }^{32} \mathrm{P}$ labeled probe overnight before exposure to BioMax MS-1 Autoradiography Film (Kodak, Rochester, NY).

\section{Northern blot analysis}

Total RNA was isolated using Trisure (Bioline, CA) from founder APP21 + PS1 and APP21 transgenic rats. Brain, heart, kidney, liver, and lung tissues were used in Northern blot analysis. Total RNA was size-separated through $1 \%$ agarose gel before transferring to the Genescreen plus membrane overnight. The membrane was prehybridized in $10 \%$ dextran sulfate, $5 \times \mathrm{SSPE}, 50 \%$ formamide, $5 \times$ Denhardt's, $1 \%$ SDS at $42{ }^{\circ} \mathrm{C}$ for $6 \mathrm{~h}$ prior to hybridization with the PS1 probe overnight. After exposure to BioMax MS-1 Autoradiography Film, membranes were stripped to remove labeled cDNA probe by boiling for $1 \mathrm{~h}$ in $1 \%$ SDS and $0.1 \times$ SSC. The intensity of the bands was determined using Kodak 1D v 3.6.3 software (New Haven, CT).

\section{Behavioral measures}

Behavioral assessment was conducted using APP + PS1 $(\mathrm{n}=12)$, APP $(\mathrm{n}=11)$, and non-transgenic Fischer 344 $(\mathrm{n}=12)$ rats when their average age was approximately 10 months. Prior to behavior studies, each rat was handled at least 3 times to reduce stress due to handling during the behavioral testing.

\section{Barnes maze}

The maze consisted of a grey circular platform $122 \mathrm{~cm}$ in diameter. The platform was surrounded by a wall that was $30.5 \mathrm{~cm}$ in height. The maze was elevated $83.8 \mathrm{~cm}$ above the floor by a stand. Twenty holes measuring $10.2 \mathrm{~cm}$ in diameter were evenly spaced around the perimeter. A rectangular grey escape box $(28 \mathrm{~cm}$ in length $\times 12.7 \mathrm{~cm}$ wide $\times 7.6 \mathrm{~cm}$ high at the area closest to the maze tapering to $16.5 \mathrm{~cm}$ high) could be placed beneath any hole. The escape box included an entry ramp that provided easy entry access for the rat. Black curtains were hung around the maze and above the maze walls to surround the apparatus and ensure that rats could only use the visual cues provided in the maze, rather than the distal cues within the testing room. Proximal cues were more likely to remain constant, within subjects and across subjects, during the course of training. Four visual cues consisting of various shapes (triangle, square, circle, cross) were placed at evenly spaced intervals on the inside of the maze walls. Two 86-W, 120-V floodlights producing 1690 lumins were hung above the platform served to brightly light the maze in order to create a potentially aversive environment to help motivate the rats to escape from the brightly lit, open surface in favor of the dark environment of the escape box. One light hung 68.5 on side of the maze while the other hung $137 \mathrm{~cm}$ from the other side.

Each rat was assigned an escape hole number under which the escape box was placed on each test trial; assigned hole numbers were alternated across rats to eliminate odor cues for consecutively tested rats. The escape box location remained constant for any individual rat across test trials. Behavioral testing consisted of two pretraining trials on the first day, and 6 evaluation trials ( 2 trials/day) over a period of 3 days. Each day, the animals were transferred in their home cage from their colony room to the testing room $30 \mathrm{~min}$ prior to the start of testing. On the pretraining trial, the rat was preexposed to the goal box (with the goal box placed in the same hole that would be used during subsequent training) by placing the rat in the goal box for $90 \mathrm{~s}$. The rats' escape from the goal box was prevented by covering the opening to the maze surface with a grey, opaque start box (a $23 \mathrm{~cm} \times 23 \mathrm{~cm}$ box). A training trial began by placing the rat under the start box positioned in the center of the platform. After $30 \mathrm{~s}$, the box was lifted and the rat had a maximum of $5 \mathrm{~min}(300 \mathrm{~s})$ to locate and enter the escape box. Latency (time it took for the rat to find and enter the escape box) and total errors (nose-pokes into non-escape holes) were recorded. If the rat did not enter the escape box within $5 \mathrm{~min}$, it was gently guided there by the experimenter's hand. After $30 \mathrm{~s}$, the rat was removed from the escape box and returned to its home cage. Rats were allowed to rest in their home cage in the testing room for $30 \mathrm{~min}$ before starting their second daily trial. The platform and escape box were cleaned after every trial with a $20 \%$ ethanol solution. After the third day, testing abated for 3 days, after which retention was evaluated for two additional trials (1 day), conducted exactly like those just described. The following day, the rats were given reversal training in which the rats were given training exactly like those of original acquisition, except that the escape box was located in a new location (in the opposite quadrant). Reversal training continued for a total of 3 days.

\section{Histopathology}

Nine of the female rats [ 3 of each: APP + PS1, APP, and wild type (WT)] were euthanized at 18-19 months of age and their brains were collected. Tissues were fixed in $10 \%$ formalin for $48 \mathrm{~h}$ prior to paraffin embedding. Hematoxylin and eosin staining was used to determine histological changes. The stained slides were analyzed using a Nikon Eclipse E600 microscope (Melville, NY) and an Olympus DP72 Camera (Center Valley, PA). 


\section{Statistical analysis}

Statistical analysis for PS1 mRNA expression, serum A $\beta$ level comparisons, and behavioral analyses were performed using general linear models in SAS (SAS 9.3, SAS Institute Inc., Cary, NC, USA). For the Barnes maze, the average of each animal's daily score was used in the analysis of overall performance during acquisition and reversal training. Comparisons of daily performance were analyzed separately and differences were considered significant at $p<0.05$ for all analyses.

\section{Results}

\section{APP21-PS1 double transgenic founders}

Forty-five rats were born from the lentiviral vector which contained PS1 transgene-injected embryos and 32 of them were PCR positive for the PS1 transgene. Southern blot results showed that transgene copy number ranged between 1 and 5 for each PS1 transgenic founder rat (Fig. 1a). Serum $A \beta_{42}$ ranged between 25 and $479 \mathrm{pg} / \mathrm{ml}$ in the founder animals at 4 weeks of age and transgene copy number positively correlated with serum AB42 levels in founder animals $(r=.54)$. Serum $A \beta_{40}$ and $A \beta_{42}$ levels at 8 weeks of age were also determined in founder animals (Fig. 1b). Due to the segregation of multiple integration sites in subsequent generations of transgenic animals, founder animals with one copy transgene were used for breeding. Founders with one PS1 transgene copy and relatively high serum $A \beta_{42}$ levels were considered in order to determine the ultimate founder animal. Founder 964 had one of the highest serum $A \beta_{42}(196 \mathrm{pg} / \mathrm{ml})$ levels as well as $A \beta_{42} / A \beta_{40}$ levels $(0.28)$ at 8 weeks of age. Furthermore, the F1 generation from founder 964 had the highest serum $A \beta_{42}$ levels and had a relatively largesized litter. Thus, founder 964 was used as the transgenic founder for generation of APP + PS1 transgenic rats.

Northern blot analysis of various tissues from the founder APP + PS1 transgenic animals showed that brain, heart, kidney, liver, and lung had 38, 8, 19, 15, and 15 fold more PS1 mRNA $(\mathrm{P}<0.01)$, respectively, compared to the APP21 rat line. Serum $A \beta_{40}$ and $A \beta_{42}$ levels at 8 weeks were positively correlated with PS1 mRNA levels in APP + PS1 founders (Table 1). Statistical analysis of the PS1 expression of founder animals was done by grouping the animals based on their 8 wk serum $A \beta_{42}$ levels. The groups comprised of rats with $A \beta 42$ serum levels of less than $100 \mathrm{pg} / \mathrm{ml}(<100)$, between 100 and $149 \mathrm{pg} / \mathrm{ml}$ (100-149), between 150 and $200 \mathrm{pg} / \mathrm{ml}$ (150200), and greater than $200 \mathrm{pg} / \mathrm{ml}$ (>200), as well as APP21 rats that did not have the PS1 transgene. PS1 expression in tissues increased significantly $(p<0.05)$ as serum $\mathrm{A} \beta_{42}$ levels increased (Fig. 2; Additional file 1: Table 1) with the exception of the liver, in which PS1 levels were not significantly different.

\section{Subsequent generations of APP + PS1 double transgenic rat line}

The APP21 line has been previously bred as a homozygous transgenic line. However, homozygous APP + PS1 rats failed to produce pups. Thus, the APP + PS1 double transgenic line was retained as homozygous for APP transgene and hemizygous for PS1 transgene.

Serum $A \beta_{40}$ and $A \beta_{42}$ levels were determined in F1 generation pups at 30 days of age and in F2 generation pups at 50 days of age (Fig. 3; Additional file 2: Table 2). The $A \beta_{42}$ levels increased with age in both APP and APP + PS1 lines and both F1 and F2 generation $\mathrm{APP}+\mathrm{PS} 1$ rats had significantly greater $(p<0.05) \mathrm{A} \beta_{42}$ levels compared to APP rats. Serum $A \beta_{42} / A \beta_{40}$ levels were approximately two times greater in the APP + PS1 rats compared to the APP rats for both F1 and F2 generations $(p<0.001)$, but serum $\mathrm{A} \beta_{40}$ levels were similar between PS1 + APP and APP rats and did not change with age.

\section{Behavior changes among AP + PS1, PS and fischer wild type rats \\ Barnes maze}

There were no statistical differences in performance between male and female rats, thus the data were collapsed over sex for all of the behavioral analyses.

Acquisition Overall, APP + PS1 rats made significantly more errors compared to WT rats $(p=0.04)$ during acquisition training, but there were no group differences in latency ( $p>0.05$; Fig. 4a). More specifically, there were no statistical differences in performance among the groups on acquisition days 1 and 2 ( $p$ s > 0.05), however, on day 3 , APP + PS1 rats made significantly more errors compared to both APP and WT rats $(p<0.001)$ and had significantly longer latencies compared to WT rats only $(p=0.02$; Fig. $4 d)$. Overall, these results suggest that the APP + PS1 rats had a larger learning deficit in the Barnes maze task than the APP rats.

Retention After the retention interval, APP + PS1 rats made numerically more errors $(p=0.1)$ and had significantly longer latencies $(p=0.01)$ than both the APP and WT rats (Fig. 4b), implying a larger memory deficit in the $\mathrm{APP}+\mathrm{PS} 1$ rats. These differences in memory retention may be due to the larger learning deficit in APP + PS1 animals during acquisition training.

Reversal Overall, APP + PS1 rats made significantly more errors $(p=0.04)$ and had significantly longer latencies $(p=0.03)$ compared to both the WT and APP rats during reversal training (Fig. 4c). More specifically, there were no significant differences on days 1 and 2 of reversal 

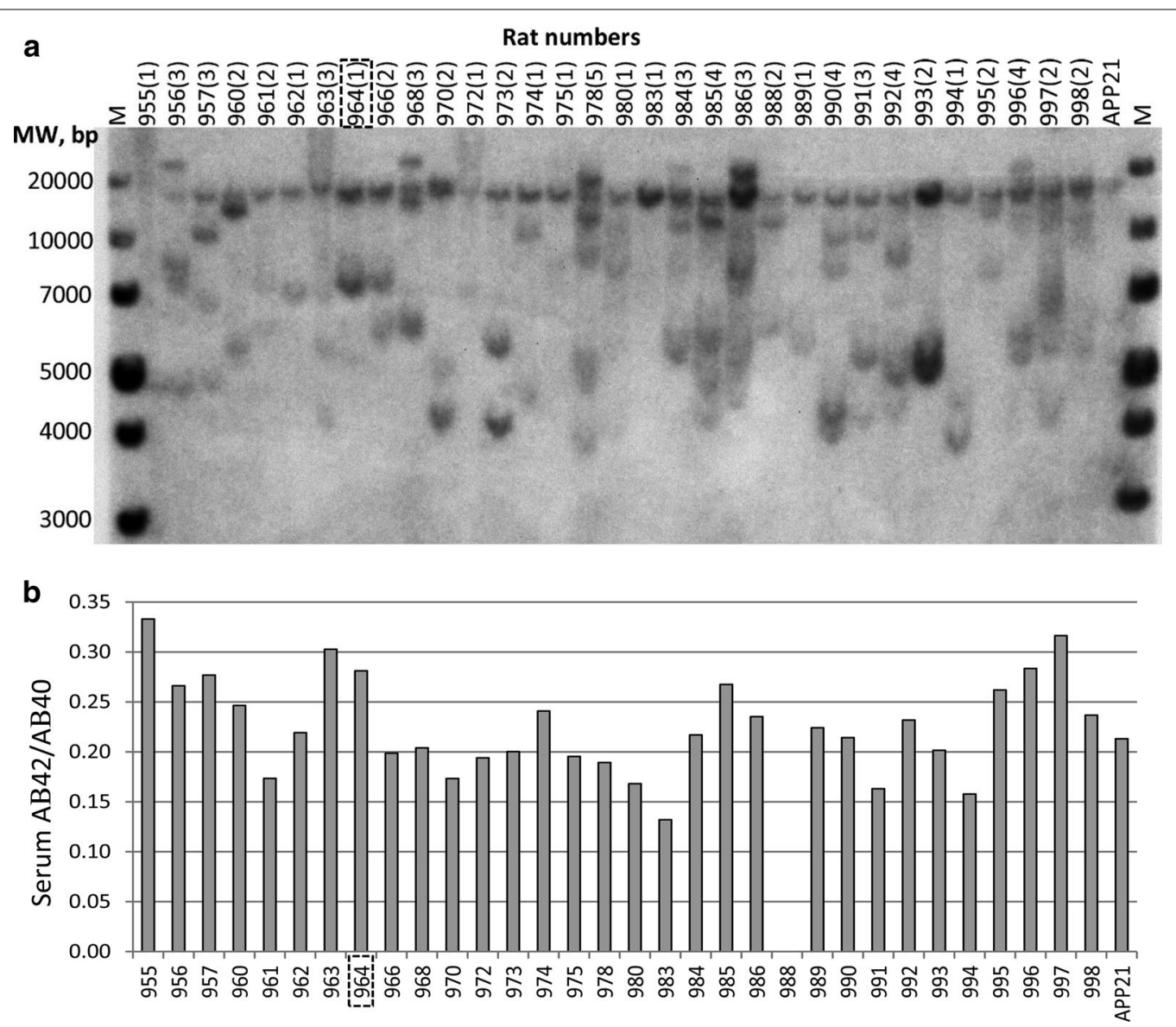

Fig. 1 a Southern blot analysis of the genomic DNA of the founder animals used to determine the copy number of PS1 transgene. The copy number of the PS1 transgene was determined by the number of hybridized genomic DNA segments in the autoradiogram and is shown in parenthesis above the rat numbers. $M$ represents the molecular weight (MW) markers. $\mathbf{b}$ Serum $A \beta_{42} / A \beta_{40}$ levels of APP + PS1 double transgenic founder animals and APP21 rats at 8 weeks of age. The $A \beta_{42} / A \beta_{40}$ ratio for rat 988 could not be determined because its $A \beta_{42}$ level was lower than the detection limit of the test. Founder 964 was selected based on having one PS1 transgene and high $A \beta_{42} / A \beta_{40}$ levels as well as for having a large litter with high $A \beta_{42} / A \beta_{40}$ levels

Table 1 Correlation coefficients of serum $A \beta_{42}$ and $A \beta_{40}$ levels to PS1 mRNA levels of the organs

\begin{tabular}{lll}
\hline PS1 $\mathbf{m R N A}$ & Serum $\mathbf{A}_{\mathbf{4 2}}$ & Serum $\mathbf{A} \boldsymbol{\beta}_{\mathbf{4 0}}$ \\
\hline Brain & 0.49 & 0.36 \\
Kidney & 0.48 & 0.30 \\
Heart & 0.60 & 0.34 \\
Liver & 0.45 & 0.22 \\
Lung & 0.54 & 0.31 \\
\hline
\end{tabular}

training $(p s>0.05)$, but on day 3, APP + PS1 rats made significantly more errors $(p<0.01)$ and had significantly longer latencies $(p=0.04)$ than both the APP and WT rats (Fig. 4e). These results suggest deficits in reversal learning in the APP + PS1 rats. Additional file 3: Table 3 contains the dataset of the Barnes Maze.

\section{Histopathology of brain}

The hippocampal pyramidal cell layer of Cornu Ammonis (CA) 1 and CA2 regions of the APP transgenic rats contained many necrotic neurons and neurofibrillary tangles (Fig. 5a, d, and g). Mineralization was also evident as indicated by the rough cell membrane observed in some of the neurons in the hippocampus and the cortex in both the APP and the APP + PS1 rats. In addition to neurons containing flame-shaped neurofibrillary tangles, hippocampus also contained remnants of neurofibrillary tangles that were embedded in the neuropil (ghost tangles) in the APP rats (Fig. 5d, g). Neurofibrillary tangles were present in viable cells that had nuclei as well as neurons with no visible nuclei (Fig. 5). The CA3 region also contained necrotic neurons and neurofibrillary tangles in both the APP and APP + PS1 rats (Fig. 5j, k).

Both the APP and APP + PS1 rats had fewer pyramidal neurons in the CA3 region compared to WT, but 


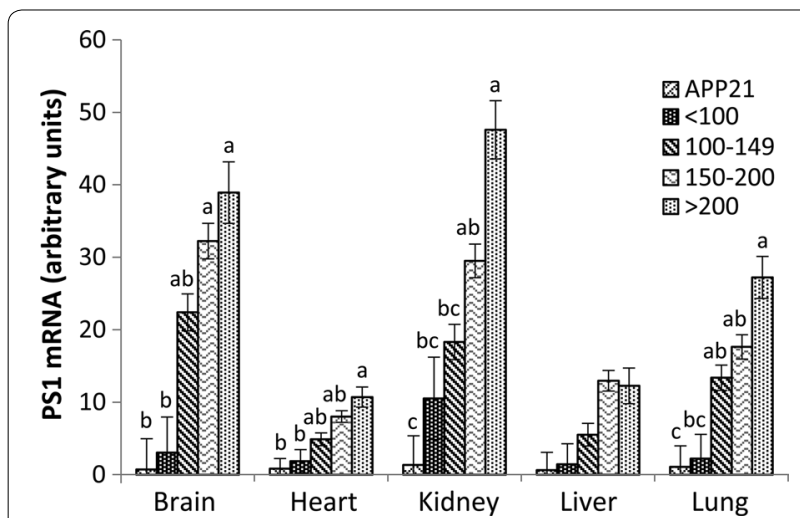

Fig. 2 PS1 expression in founder animals and APP controls as determined by Northern blot analysis. The expression of PS1 was analyzed by combining animals with similar serum $A \beta_{42}$ levels and statistical analysis conducted for each tissue. The figure legend indicates the range of serum $A \beta_{42}$ level $(\mathrm{pg} / \mathrm{ml})$ of the founder animals or the APP control rats. Different letters above each bar indicates significantly different $(p<0.05)$ expression

the APP + PS1 animals had more severe neuronal loss in the CA3 region (Fig. 5b). The hippocampal pyramidal cell layer in the APP + PS1 rats had many neurons with central and segmental chromatolysis, showing eccentric displacement of the nucleus and loss of Nissl substance with the exception of the margins of the cell body (Fig. 5h, k). Both the APP and the APP + PS1 brain cortex contained neurons with various degrees of degeneration that ranged from neurons with condensed Nissl substance to dark necrotic neurons and ghost tangles (Fig. 5p, q). The brain cortex of the APP + PS1 animals had larger areas with dark stained neurons, which indicated more severe neuronal necrosis (Fig. 5q).

\section{Discussion}

The production of transgenic rats using lentiviral vectors is an efficient method for gene transfer. However, the major drawback of lentiviral vectors is the integration of the transgene into multiple sites of the genome. Multiple copies of the transgene result in high levels of expression, but segregation in subsequent generations drastically decreases transgene expression. In this study, the founder animal was selected among the animals that had only one copy of the transgene and those that also had the greatest serum $A \beta_{42}$ levels and $A \beta_{42} / A \beta_{40}$ ratio.

Serum $A \beta_{42}$ and $A \beta_{40}$ levels were positively correlated with PS1 mRNA expression, indicating that the addition
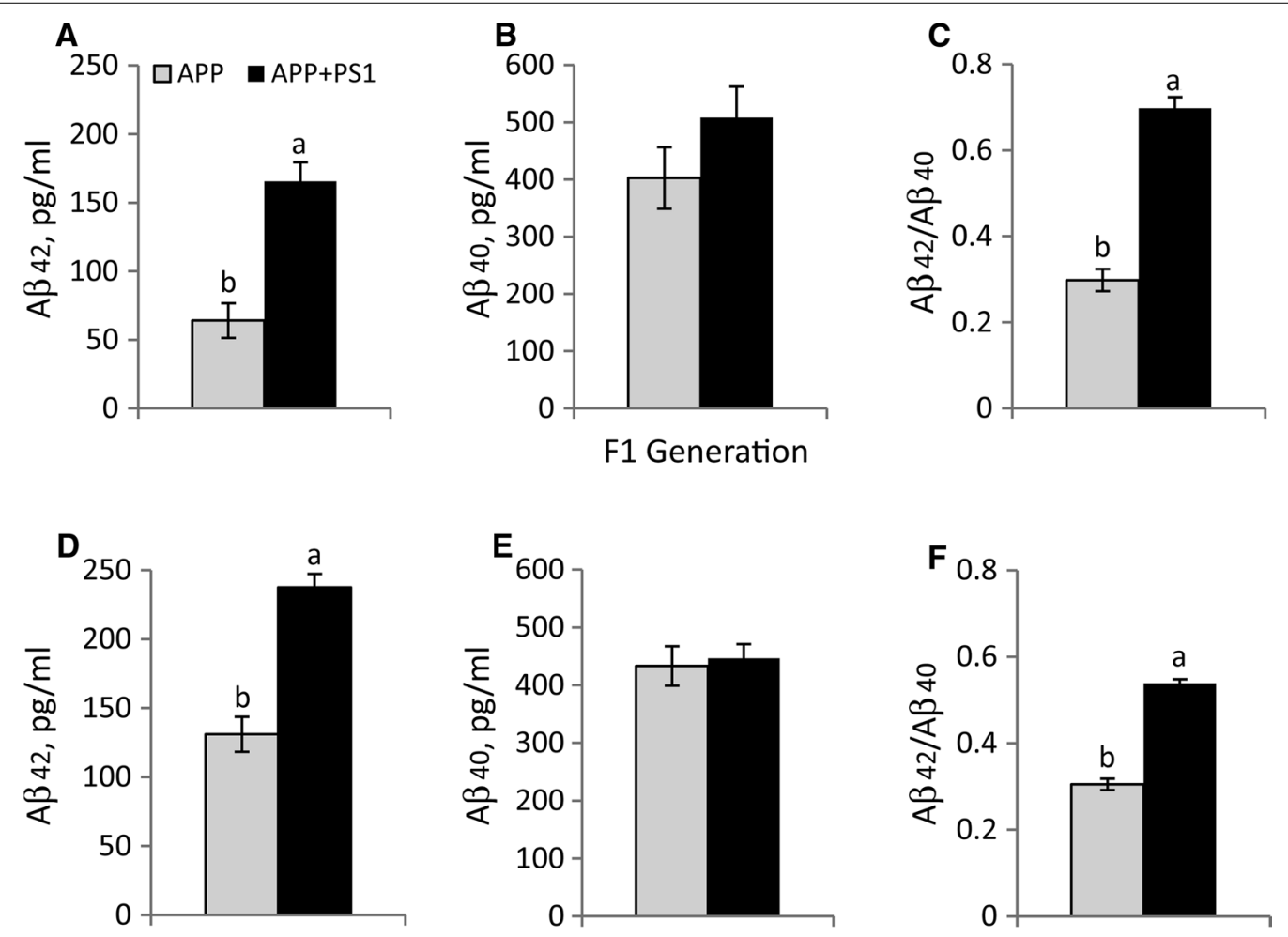

F2 Generation

Fig. 3 Serum $A \beta_{42}(\mathbf{A}, \mathbf{D}), A \beta_{40}(\mathbf{B}, \mathbf{E})$ and $A \beta_{42} / A \beta_{40}(\mathbf{C}, \mathbf{F})$ levels of 30 day old $\mathrm{F} 1$ and 50 day old $\mathrm{F} 2$ generations. Different letters above each bar indicate significantly different levels of $A \beta_{42}$ and $A \beta_{42} / A \beta_{40}$ 

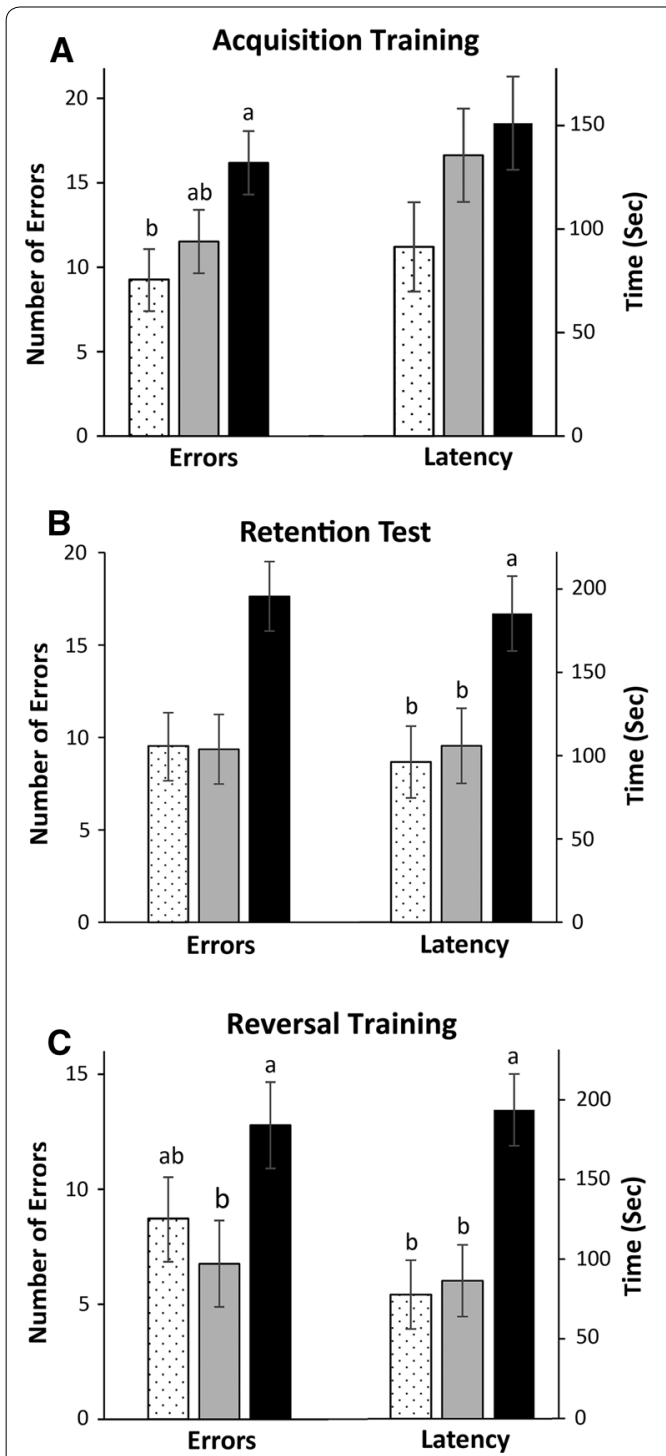



$\square W T \quad \square$ APP $\square$ APP+PS1

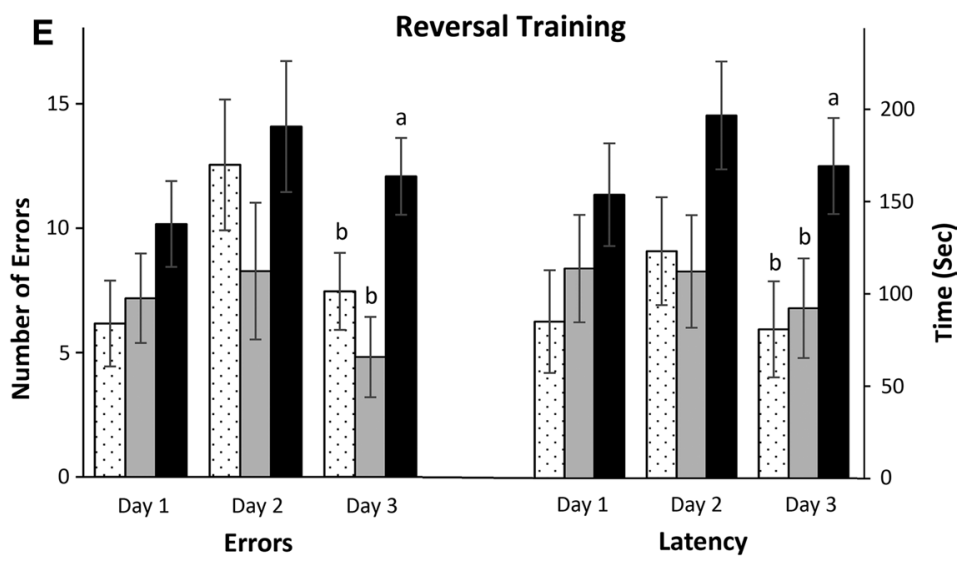

Fig. 4 Overall performance of Barnes maze Acquisition Training (A) Retention (B), and Reversal Training (C). Daily performance of Acquisition Training $(\mathbf{D})$, and Reversal Training (E). Data is expressed as group mean \pm SEM. Different letters above each bar indicates significantly different $(p<0.05)$ expression

of the PS1 transgene contributed to the production of both $A \beta_{42}$ and $A \beta_{40}$. Furthermore, the greater correlation efficiencies for $A \beta_{42}$ and PS1 mRNA expression indicate preferential cleavage of $\mathrm{A} \beta_{42}$ by the PS1 enzyme. Transfection using PS1 resulted in an approximately 1.6 fold increase in $A \beta_{42} / A \beta_{40}$ levels [16] in cell cultures. Similarly, the APP + PS1 transgenic animals had approximately a twofold $A \beta_{42} / A \beta_{40}$ increase compared to the APP rats. The level of PS1 mRNA expression varied by tissue with the highest PS1 expression in the brain and kidney, while the heart and liver had lower expression. Tissue specific expression was also observed in the APP transgenic rats [1].
Histologic examination of the brains showed that both the APP and the APP + PS1 animals exhibited pathological abnormalities typically associated with AD. Interestingly, the APP rats had large hippocampal sections containing neurons with neurofibrillary tangles. The hippocampus of the APP + PS1 rats also contained neurofibrillary tangles, but these were more sporadic compared to what was observed in the APP rats. Interestingly, neuronal loss was much greater in the CA3 region of the APP + PS1 rats. Another difference between the hippocampus in APP and the APP + PS1 animals was that neurons in the CA1 and the CA2 regions of the APP + PS1 animals had chromatolysis. Chromatolysis is 


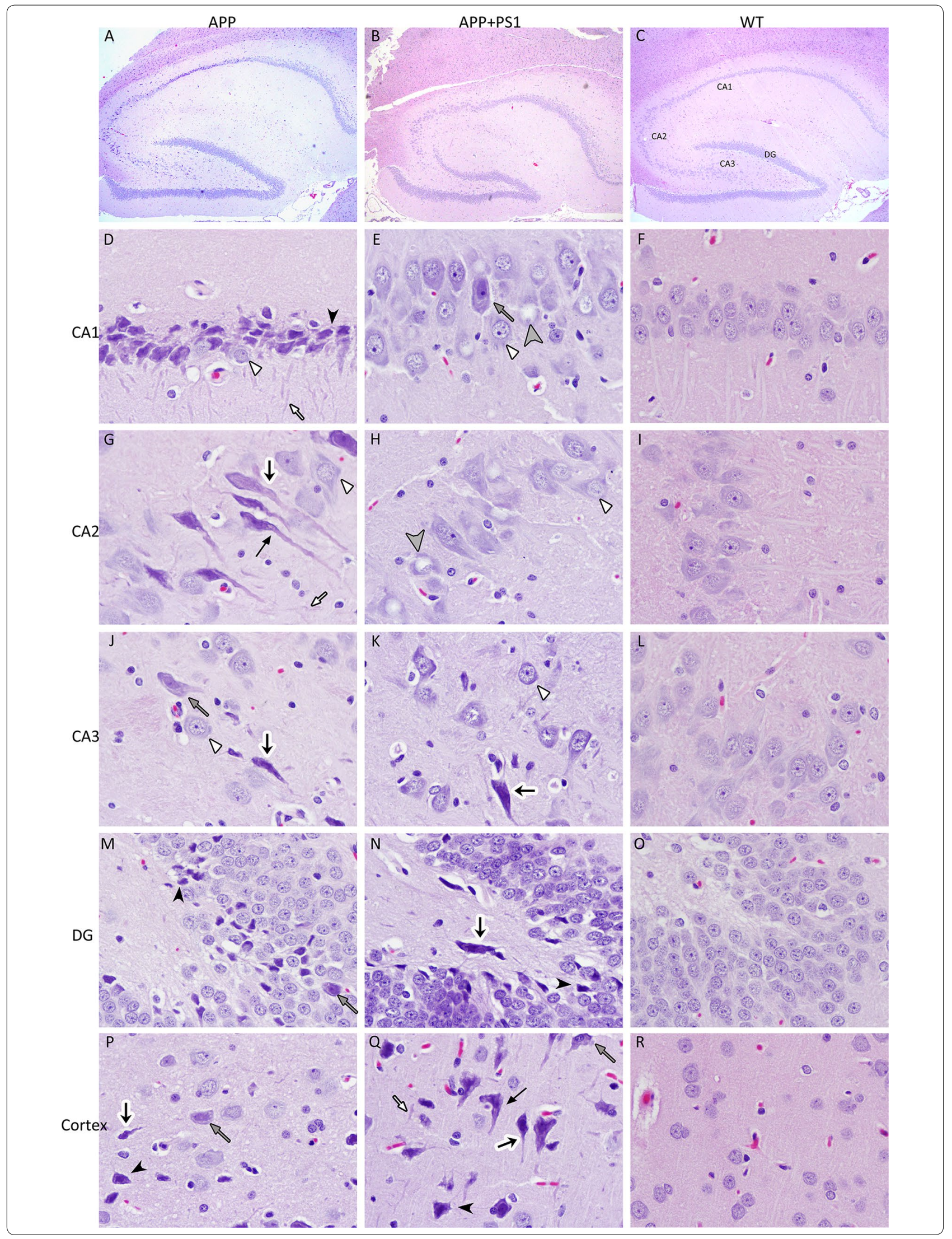


Fig. 5 Hematoxylin and Eosin staining of APP, APP + PS1, and WT rat brains. 4x magnification of a hippocampus section from an APP (a), APP + PS1 (b), and WT rat (c). CA1, CA2, CA3 regions of the hippocampus and dentate gyrus (DG) are labeled in c. Panels $\mathbf{d}-\mathbf{r}$ show $60 \mathrm{X}$ magnification of the brain and each arrow style represents similar pathology throughout the figure. $\mathbf{d}$ APP CA1 region with necrotic and dark colored pyramidal neurons (black arrowhead), ghost tangles (white arrow), and normal looking neurons (white arrow head). e APP + PS1 CA1 showing degeneration with condensed Nissl substance (grey arrow) and chromatolysis (grey arrow head). fWT CA1. g APP CA2 with neurofibrillary tangles in viable neurons with visible nuclei (black arrow) and non-viable neurons (black arrow with white outline) and ghost tangles. h APP + PS1 CA2 showing chromatolysis in neurons. i WT CA2. j APP CA3 with neurofibrillary tangles and condensed Nissl substance. $\mathbf{k}$ APP + PS1 CA3 with neurofibrillary tangles. IWT CA3. $\mathbf{m}$ APP DG granule cells with condensed Nissl substance and necrotic cells. $\mathbf{n}$ APP + PS1 DG with necrotic cells and neurofibrillary tangles. $\mathbf{o}$ WT DG. p, q APP and APP + PS1 cortex neurons with condensed Nissl substance, neurofibrillary tangles, necrotic neurons, and ghost tangles. $\mathbf{r}$ WT cortex

caused by axonal injury in motor neurons [18] and it has been associated with neurotoxicity and $\mathrm{AD}[5,15,22]$, but it is not reported as widely as the presence of neurofibrillary tangles or $\mathrm{A} \beta$ plaques.

A previously reported rat model for $\mathrm{AD}$, the $\mathrm{TgF} 344$ AD model [6], was found to produce plaques at 15 months. The difference in plaque production between the TgF344-AD and the APP + PS1 rats could be due to the fact that the PS1 transgene in the TgF344-AD had $\Delta$ exon 9 mutation, as opposed to the L166P mutation form of the PS1 transgene that was used in these APP + PS1 rats. On the other hand, the behavioral characterization of the APP + PS1 rats in this current study revealed learning and memory deficits similar to what has been found in TgF344-AD rats. In addition, previous research has found that an $\mathrm{AD}$ mouse model that did not develop plaques even as late as 30 months old also showed memory deficits [9]. Consequently, the presence of $A \beta$ plaques may not always correlate with AD severity. For example, Shankar et al. [21] showed that $A \beta$ dimers are synaptotoxic and disrupted memory, whereas insoluble $A \beta$ plaque cores from Alzheimer's diseased cortex did not impair long-term potentiation. Furthermore, the number of $A \beta$ plaques in the brain has not been found to correlate with the severity of cognitive impairments in humans or in APP and/or PS transgenic mice [3].

Wild type rats outperformed both the APP and the APP + PS1 rats during the initial learning phase of the Barnes maze test. Additionally, retention of the learned task was poorer in the APP + PS1 rats relative to both the APP and the WT rats. As stated earlier, one of the major differences between the APP + PS1 and the APP rats was that the APP + PS1 rats had neuronal loss in the CA3 region of hippocampus (Fig. 5b shows an APP + PS1 rat with very few neurons in the CA3 region). The CA1 region recodes information from the $\mathrm{CA} 3$ region and sets up associatively learned backprojections to the neocortex to allow subsequent retrieval of information to the neocortex [13]. The hippocampal CA3 region and the dentate gyrus also play an important role in the encoding of new spatial information and processing the geometry of the environment [12], which are both crucial to the successful completion of the task. Thus, the differences between the APP + PS1, APP, and WT rats in learning and retention in the task may be related to the differences in the pathology of the hippocampus.

\section{Conclusions}

The addition of the PS1 transgene to the APP transgenic rat genome affected serum $A \beta_{42}$ and $A \beta_{42} / A \beta_{40}$ levels, brain histopathology, as well as, the cognitive behavior of the double transgenic rats. Although the APP rat brain had many histopathogical abnormalities that are often seen in AD brains, their memory did not show significant impairment during the behavioral testing. In contrast, the APP + PS1 rats showed significantly reduced memory retention compared to the APP and the WT rats, in addition to, neuronal loss and necrosis in the hippocampus and brain cortex.

\section{Additional files}

Additional file 1: Table 1. contains dataset for Northern blot analysis (see Fig. 2). PS1 mRNA level, serum $A \beta 42$ and tissue are reported in the table.

Additional file 2: Table 2. contains dataset for serum $A \beta_{42}, A \beta_{40}$ and $A \beta_{42} / A \beta_{40}$ ratio of $F 1$ and $F 2$ generation transgenic rats (see Fig. 3 ).

Additional file 3: Table 3. contains dataset for Barnes maze (see Fig. 4). The table includes acquisition day 1,2 and 3 latency and errors and their averages, retention latency and errors and reversal days 1, 2 and 3 latency and errors and their averages.

\section{Abbreviations}

AD: alzheimer's disease; AB: amyloid-beta; APP: amyloid precursor protein; CA: cornu Ammonis; eGFP: enhanced green fluorescence protein; IP: intraperitoneal; pLV: lentivial vector; PMSG: pregnant mares' serum gonadotropins; PS1: presenilin 1; SSC: saline sodium citrate; SDS: sodium dodecyl sulfate; Ubi-C: ubiquitin-C; WT: wild type; WRE: woodchuck hepatitis virus post-transcriptional regulatory element.

\section{Authors' contributions}

CA was responsible for genotypic and phenotypic characterization of transgenic rats, statistical analysis of data, colony management, and writing the paper. JJ generated lentiviral vectors and performed ELISA. DK and TRS have conducted rat behavioral assessment and analysis. YA and AWC generated transgenic rats. YA designed the research, and contributed to the analysis of the data. All authors read and approved the final manuscript.

\section{Author details}

${ }^{1}$ Department of Veterinary Pathobiology, College of Veterinary Medicine, University of Missouri, 1600 East Rollins Street, Room W191, Columbia, MO 65211, USA. ${ }^{2}$ Department of Psychological Sciences, University of Missouri, Columbia, 
MO 65211, USA. ${ }^{3}$ Yerkes National Primate Research Center, Emory University, Atlanta, GA 30329, USA. ${ }^{4}$ Department of Neurology, Center for Neurodegenerative Disease, Emory University, Atlanta, GA 30322, USA.

\section{Acknowledgements}

This study was funded by a grant from Alzheimer Research Consortium and the University of Missouri startup funds. The authors would also like to thank Dr. Marcia Hart for helping assess brain histopathology.

\section{Competing interests}

The authors declare that they have no competing interests.

\section{Availability of data and materials}

Datasets are submitted as supplementary material.

\section{Ethics approval and consent to participate}

All animal studies were approved by University of Missouri's Animal Care and Use Committee (Protocol Number 7995).

Received: 21 April 2016 Accepted: 27 June 2016

Published online: 07 July 2016

\section{References}

1. Agca C, Fritz JJ, Walker LC, Levey Al, Chan AW, Lah JJ, Agca Y. Development of transgenic rats producing human beta-amyloid precursor protein as a model for Alzheimer's disease: transgene and endogenous APP genes are regulated tissue-specifically. BMC Neurosci. 2008;9:28.

2. Alzheimer's Association. 2015 Alzheimer's disease facts and figures. Alzheimers Dement 2015; 11(3):332-84.

3. Benilova I, Karran E, De Strooper B. The toxic A $A$ oligomer and Alzheimer's disease: an emperor in need of clothes. Nat Neurosci. 2012;15(3):349-57.

4. Burdick D, Soreghan B, Kwon M, Kosmoski J, Knauer M, Henschen A, Yates J, Cotman C, Glabe C. Assembly and aggregation properties of synthetic Alzheimer's A4/beta amyloid peptide analogs. J Biol Chem. 1992:267(1):546-54.

5. Cataldo AM, Hamilton DJ, Nixon RA. Lysosomal abnormalities in degenerating neurons link neuronal compromise to senile plaque development in Alzheimer disease. Brain Res. 1994;640(1-2):68-80.

6. Cohen RM, Rezai-Zadeh K, Weitz TM, Rentsendorj A, Gate D, Spivak I, Bholat Y, Vasilevko V, Glabe CG, Breunig JJ, Rakic P, Davtyan H, Agadjanyan MG, Kepe V, Barrio JR, Bannykh S, Szekely CA, Pechnick RN, Town T. A transgenic Alzheimer rat with plaques, tau pathology, behavioral impairment, oligomeric aß, and frank neuronal loss. J Neurosci. 2013;33(15):6245-56.

7. De Strooper B. Aph-1, Pen-2, and Nicastrin with Presenilin generate an active gamma-Secretase complex. Neuron. 2003;38(1):9-12.

8. Deng Y, Tarassishin L, Kallhoff V, Peethumnongsin E, Wu L, Li YM, Zheng H. J Neurosci. 2006;26:3845-54.
9. Gandy S, Simon AJ, Steele JW, Lublin AL, Lah JJ, Walker LC, Levey Al, Krafft GA, Levy E, Checler F, Glabe C, Bilker WB, Abel T, Schmeidler J, Ehrlich ME. Days to criterion as an indicator of toxicity associated with human Alzheimer amyloid-beta oligomers. Ann Neurol. 2010;68(2):220-30.

10. Jarrett JT, Berger EP, Lansbury PT Jr. The C-terminus of the beta protein is critical in amyloidogenesis. Ann N Y Acad Sci. 1993;695:144-8.

11. Kandimalla RJ, Wani WY, Binukumar BK, Gill KD. siRNA against presenilin 1 (PS1) down regulates amyloid $\beta 42$ production in IMR-32 cells. J Biomed Sci. 2012;19:2.

12. Kesner RP. Behavioral functions of the CA3 subregion of the hippocampus. Learn Mem. 2007;14(11):771-81.

13. Kesner RP, Rolls ET. A computational theory of hippocampal function, and tests of the theory: new developments. Neurosci Biobehav Rev. 2015;48:92-147.

14. Kim J, Onstead L, Randle S, Price R, Smithson L, Zwizinski C, Dickson DW, Golde T, McGowan E. Abeta40 inhibits amyloid deposition in vivo. J Neurosci. 2007;27(3):627-33.

15. Kotariya NT, Bikashvili TZ, Zhvaniya MG. Chkhikvishvili TsG. Ultrastructure of hippocampal field CA1 in rats after status epilepticus induced by systemic administration of kainic acid. Neurosci Behav Physiol. 2010;40(2):127-30.

16. Li N, Liu K, Qiu Y, Ren Z, Dai R, Deng Y, Qing H. Effect of Presenilin Mutations on APP Cleavage; insights into the pathogenesis of FAD. Front Aging Neurosci. 2016;8:51.

17. Lois C, Hong EJ, Pease S, Brown EJ, Baltimore D. Germline transmission and tissue-specific expression of transgenes delivered by lentiviral vectors. Science. 2002;295:868-72.

18. Mcllwain DL, Hoke VB. The role of the cytoskeleton in cell body enlargement, increased nuclear eccentricity and chromatolysis in axotomized spinal motor neurons. BMC Neurosci. 2005;6:19.

19. Masters CL, Selkoe DJ. Biochemistry of amyloid beta-protein and amyloid deposits in Alzheimer disease. Cold Spring Harb Perspect Med. 2012;2(6):a006262.

20. Rosen RF, Fritz JJ, Dooyema J, Cintron AF, Hamaguchi T, Lah JJ, LeVine $\mathrm{H}$ 3rd, Jucker M, Walker LC. Exogenous seeding of cerebral $\beta$-amyloid deposition in $\beta$ APP-transgenic rats. J Neurochem. 2012;120(5):660-6.

21. Shankar GM, Li S, Mehta TH, Garcia-Munoz A, Shepardson NE, Smith I, Brett FM, Farrell MA, Rowan MJ, Lemere CA, Regan CM, Walsh DM, Sabatini BL, Selkoe DJ. Amyloid-beta protein dimers isolated directly from Alzheimer's brains impair synaptic plasticity and memory. Nat Med. 2008;14(8):837-42.

22. Sil S, Goswami AR, Dutta G, Ghosh T. Effects of naproxen on immune responses in a colchicine-induced rat model of Alzheimer's disease. NeurolmmunoModulation. 2014;21(6):304-21.

23. Walsh DM, Selkoe DJ. A beta oligomers-a decade of discovery. J Neurochem. 2007;101(5):1172-84.

\section{Submit your next manuscript to BioMed Central and we will help you at every step:}

- We accept pre-submission inquiries

- Our selector tool helps you to find the most relevant journal

- We provide round the clock customer support

- Convenient online submission

- Thorough peer review

- Inclusion in PubMed and all major indexing services

- Maximum visibility for your research

Submit your manuscript at www.biomedcentral.com/submit
(O) BioMed Central 